\title{
DNAJC12 as a Mediator Between ESR1 and ERBB4 in Breast Carcinoma Cells
}

\author{
Mianjie Lin ${ }^{1 \dagger}$, Ya-Nan Wang $^{2 \dagger}$, Yixin Ye ${ }^{2}$, Zhelei Xiong ${ }^{2}$, Fengbiao Guo ${ }^{2}$ \\ and Haibin Chen ${ }^{1,2 *}$ \\ 1 Guangdong Provincial Key Laboratory of Breast Cancer Diagnosis and Treatment, Shantou University Medical College, \\ Shantou, China, ${ }^{2}$ Department of Histology and Embryology, Shantou University Medical College, Shantou, China
}

Mutation of the DNAJC12 gene is typically associated with non-progressive Parkinsonism, but is also detectable in breast carcinoma where its contribution and mechanisms are unexplored. In breast carcinoma, ESR1 was positively correlated with DNAJC12 and ERBB4, and DNAJC12 was positively correlated with ERBB4. We used the GEO2R tool to compare differential gene expression of MCF-7 cells, following ESR1 knockdown (GEO database, E-GEOD-27473 array), and found decreased expression of DNAJC12 and

OPEN ACCESS

Edited by:

Swee Keong Yeap,

Xiamen University, Malaysia

Reviewed by:

Yew Woh Hui,

Xiamen University, Malaysia Davoud Jazayeri,

Sunway University, Malaysia

*Correspondence: Haibin Chen

chenhb@stu.edu.cn

${ }^{\dagger}$ These authors have contributed equally to this work

Specialty section:

This article was submitted to Molecular and Cellular Oncology,

a section of the journal

Frontiers in Oncology

Received: 11 July 2020 Accepted: 05 January 2021

Published: 24 February 2021

Citation:

Lin $M$, Wang $Y-N$, Ye $Y$, Xiong Z, Guo $F$ and Chen H (2021) DNAJC12 as a Mediator Between ESR1 and ERBB4 in Breast Carcinoma Cells.

Front. Oncol. 11:582277. doi: 10.3389/fonc.2021.582277
ERBB4 in ESR1-silenced MCF-7 cells. The number of identical genes having correlativity with ESR1, DNAJC12, or ERBB4 was 12,165 (66.41\%). These results suggest that ESR1 can promote the expression of DNAJC12 and ERBB4, and DNAJC12 can enhance the expression of ERBB4 in MCF-7 cells, implying that there may be a regulatory network among these three genes.

Keywords: ESR1, DNAJC12, ERBB4, breast carcinoma, signaling pathway

\section{INTRODUCTION}

Breast carcinoma is one of the most common malignancy and predominant cause of cancer-related death in women around the world, with a one in seven lifetime risk (1-3). Breast cancer, for the majority of patients, is treated with upfront surgery followed by other adjuvant (post-operative) modalities including radiotherapy, chemotherapy, endocrine therapy, immunotherapy, and adjuvant therapy of traditional Chinese medicine (4-9). Estrogen receptor (ER)-positive and/or progesterone receptor (PR)-positive breast cancer accounts for approximately $70 \%$ of all breast cancers, and $85 \%$ of those in women over 70 years of age (10-12). ER-positive tumors often respond poorly to neoadjuvant chemotherapy and therefore require robust alternatives (13-16). So far, three categories of neoadjuvant endocrine therapy are available: selective ER modulators (primarily tamoxifen), selective ER degraders (fulvestrant), and aromatase inhibitors (including letrozole, anastrozole, and exemestane) which block estrogen synthesis (17). However, the adoption of neoadjuvant endocrine therapy for ER-positive tumors has been much slower and such treatments have serious side effects that can lead to poor quality of life for patients (18). Thus, biological targeted therapy is concerned by more and more medical workers.

Heat shock proteins (HSPs) are a class of heat stress proteins that exist widely from bacteria to mammals, many of which have molecular chaperone activity, and play an important role in the occurrence and development of cancer. HSPs are mainly induced by heat shock or other stresses to 
aid in the folding, processing, and maturation of auxiliary proteins, and can be classified into Hsp27, Hsp40, Hsp60, Hsp70, Hsp90, and larger HSPs according to molecular mass (19). Several heat shock protein genes are deregulated in all molecular subtypes of breast carcinoma while others appear deregulated in specific molecular subtypes, and the overall survival of breast carcinoma patients appears associated with the expression level of certain HSPs (20). In addition, HSPs play important roles in breast carcinoma, including regulating the status of extracellular matrix proteins (21), maintaining the survival and proliferation of carcinoma cells $(22,23)$, regulating protein expression and maintaining stability $(24,25)$, and regulating the cell cycle and migration (26). Many studies have proved that HSPs are beneficial for the diagnosis, treatments, and efficacy evaluation of cancers. HSPs may also have potential clinical uses as biomarkers for cancer diagnosis, for assessing disease progression, or as therapeutic targets for cancer therapy (27-32).

DNAJC12, also known as JDP1 or HPANBH4, is a member of the DnaJ heat shock protein family (Hsp40) C12 $(33,34)$. The mRNA expression level of DNAJC12 is generally higher in breast carcinoma (35), especially in ESR1-positive breast carcinoma tissues (36). ESR1 is associated with multiple HSPs, such as Hsp27, Hsp70, Hsp90 (37-39), and in ESR1-positive human MCF-7 breast carcinoma cells, 17ß-Estradiol can induce DNAJC12 mRNA expression (36). However, the relationship between ESR1 and DNAJC12 is still unclear, and the signaling pathway is also unknown. In this study, we explored the relation between ESR1 and DNAJC12, and predicted DNAJC12-related genes involved in signaling pathways so as to offer a preliminary theoretical basis for the expression and function of DNAJC12 in breast carcinoma, which may provide a new targeted therapy site of breast cancers.

\section{MATERIALS AND METHODS}

\section{Microarray Source}

The gene expression dataset (E-GEOD-27473) analyzed in this study was obtained from EMBL (https://www.ebi.ac.uk/), and was comprised of three profiles of MCF-7 cells with normal ESR1 expression and three profiles of MCF-7 cells with the ESR1 gene silenced (40).

\section{Plasmids}

Plasmids encoding ESR1 and DNAJC12 cDNAs, cloned into the pcDNA3.1 + vector, were purchased from the Hangzhou Yanzhen Biotechnology Co., Ltd. and extracted with a FastPure Plasmid Mini Kit (Vazyme Biotech, DC201).

\section{Analyzing of Gene Chip by GEO2R Tool}

GEO2R (https://www.ncbi.nlm.nih.gov/geo/geo2r/) is an interactive web tool that allows users to compare two or more groups of samples in order to identify genes that are differentially expressed. GEO2R tool was used to identify the differentially expressed genes in the dataset (E-GEOD-27473), and the gene expression values and adjusted p-values of genes (ESR1, DNAJC12, and ERBB4) were saved in this study.

\section{Predicting ESR1 Binding Sites by PROMO}

PROMO $(41,42)$, a virtual laboratory for the study of transcription factor binding sites in DNA sequences, was used to search for the potential binding sites of ESR1 on the promoter region of DNAJC12 in this study.

\section{Analysis of TCGA data by cBioPortal}

The cBioPortal for Cancer Genomics (http://www.cBioPortal. org/) is an online tool providing visualization, analysis, and downloading cancer genomic datasets $(43,44)$. In this study, 1,108 invasive breast carcinoma samples from the TCGA database were used to identify genes correlated with ESR1, DNAJC12, and ERBB4. According to the key genes of signal pathways provided by the cBioPortal, the VBA program was used to screen the genes having a correlation with DNAJC12 (45). Then, PCR detection was employed to verify differential expression of the identified genes. The $\mathrm{R}$ program was used to draw the Venn diagram for analyzing the distribution of those genes whose "q-value" was less than or equal to 0.05 in the lists related to the three genes.

\section{Cell Culture}

MCF-7 cells (ATCC cell bank) used in this study were kindly provided by Professor Wenxiu Ni (Department of Chemistry, Shantou University Medical College). Cells were cultured with high glucose DMEM medium (Gibco, C11995500BT) containing $10 \%$ FBS (Gibco, $16010-159$ ) at $37^{\circ} \mathrm{C}$ with $5 \% \mathrm{CO}_{2}$. MCF-7 cells were transfected with ESR1 or DNAJC12 plasmids for 0, 24, 48, and $72 \mathrm{~h}$ when cells reached $70 \sim 90 \%$ confluence.

\section{Extraction of Total Cellular RNA and PCR}

Total RNA of cultured cells was extracted using Trizol (TIANGEN, DP405). Subsequent RT-PCR was performed using a HiScriptII 1st Strand cDNA Synthesis Kit (Vazyme Biotech, $\mathrm{R} 212-02)$. Sequences of primers are listed in Table 1. PCR reaction conditions involved a pre-denaturation in $95^{\circ} \mathrm{C}$ for $3 \mathrm{~min}, 29 \mathrm{PCR}$ cycles for amplification (each consisting of $15 \mathrm{~s}$ of denaturation at $95^{\circ} \mathrm{C}, 30 \mathrm{~s}$ of annealing at $56^{\circ} \mathrm{C}$, and $30 \mathrm{~s}$ of extension at $72^{\circ} \mathrm{C}$ ), $10 \mathrm{~min}$ at $72^{\circ} \mathrm{C}$ for final extension, then stored at $4^{\circ} \mathrm{C}$. PCR products were subjected to agarose gel electrophoresis.

TABLE 1 | Base Sequence of Primers.

\begin{tabular}{|c|c|}
\hline Gene & Primer sequence $\left(5^{\prime} \rightarrow 3^{\prime}\right)$ \\
\hline \multirow[t]{2}{*}{ DNAJC12 } & F: CAGACAAGCATCCTGAAAACCC \\
\hline & R: TCGCCAGTGGTCATAGCGGGC \\
\hline \multirow[t]{2}{*}{ APH1B } & F: TCTACTGATTTCGTCCCT \\
\hline & R: TTTATAATATGCAAATCGGAAC \\
\hline \multirow[t]{2}{*}{ CCNE1 } & F: TGCCTTGAATTTCCTTATGGTA \\
\hline & R: AGTTCTCTATGTCGCACCAC \\
\hline \multirow[t]{2}{*}{ IGF1R } & F: CGCCTCCAACTTCGTCT \\
\hline & R: TCCTCAACTTGTGATCCGTA \\
\hline \multirow[t]{2}{*}{ BCL2 } & F: ACTGAGATTTCCACGCCGAAG \\
\hline & R: TTCTCGGCACAATTGGTAGCTT \\
\hline \multirow[t]{2}{*}{ ERBB4 } & F: TTACGCATTATTCGTGGGA \\
\hline & R: CAGTACAGGACTTATGGCAAC \\
\hline \multirow[t]{2}{*}{$\beta$-Actin } & F: GAGCTACGAGCTGCCTGACG \\
\hline & R: GTAGTTCGTGGATGCCACAG \\
\hline
\end{tabular}




\section{Western Blot}

MCF-7 cells were harvested, and then lysed in RIPA buffer containing PMSF (Solarbio, R0010) and proteinase inhibitors (Sangon Biotech, C600386-0001), and the lysates were clarified by centrifugation at $12,500 \mathrm{rpm}$ for $15 \mathrm{~min}$ at $4^{\circ} \mathrm{C}$. A BCA Protein Assay Kit (Beyotime Biotechnology, P0012) was used to determine protein concentration. Total cellular protein was separated by SDSPAGE and electro-transferred onto PVDF membranes (Millipore, ISEQ00010). Then the membranes were blocked with $5 \%$ milk powder (Sangon Biotech, A600669-0250) and washed by $0.1 \%$ TBS/T for $10 \mathrm{~min}$. Subsequently, the membranes were incubated with primary antibody. The following primary antibodies were used: mouse monoclonal anti-ESR1 (Santa Cruz Biotechnology, sc8002), rabbit polyclonal anti-DNAJC12 (Proteintech, 12338-1-AP), rabbit polyclonal anti-ERBB4 (Proteintech, 19943-1-AP), or mouse monoclonal anti- $\beta$-actin (Beyotime Biotechnology, AF0003) at $4^{\circ} \mathrm{C}$ overnight. After washing three times in $0.1 \% \mathrm{TBS} / \mathrm{T}$, membranes were incubated with the secondary HRP-conjugated goat antirabbit IgG $(\mathrm{H}+\mathrm{L})$ (Beyotime Biotechnology, A0208) or HRPconjugated goat anti-mouse IgG $(\mathrm{H}+\mathrm{L}$ ) (Beyotime Biotechnology, A0216). Proteins were visualized with an ECL system.

\section{Statistical Analysis}

Statistical analysis was conducted using Image J software, GraphPad Prism 7, or SPSS software version 20.0. Additionally, when the variances were homogeneous, an LSD test was applied to make pairwise comparisons. The Games-Howell test was applied to make pairwise comparisons when the variances were uneven. Differences between groups were evaluated by one-way ANOVA $\left({ }^{*}: P<0.05\right.$, ${ }^{* *}: P<0.01,{ }^{* * *}: P<0.001,{ }^{* * * *}: P<0.0001$.). Data were expressed as mean \pm SEM of at least three independent experiments. A $p$-value $<$ 0.05 was considered to be statistically significant.

\section{RESULTS}

\section{ESR1 Promotes Expression of DNAJC12}

To explore the upstream regulatory genes of DNAJC12 in MCF7 cells through GEO2R, it was shown that the mRNA level of DNAJC12 was reduced in ESR1-silenced MCF-7 cells (Tables 2 and 3). Also, ESR1 has four potential binding sites on the DNAJC12 promoter (Table $\mathbf{4}$ and Figure 1 ) at the $125^{\text {th }}$ nucleotide, $450^{\text {th }}$ nucleotide, $886^{\text {th }}$ nucleotide, or $1,034^{\text {th }}$ nucleotide. The dissimilarity indices were less than 0.000001 ,

TABLE 2 | ESR1 mRNA expression in gene chip (E-GEOD-27473).

\begin{tabular}{llr}
\hline Sample & \multicolumn{1}{c}{ Title } & Value \\
\hline GSM678802 & MCF-7, biological rep1 & 12.2408 \\
GSM678803 & MCF-7, biological rep2 & 12.2049 \\
GSM678804 & MCF-7, biological rep3 & 12.2326 \\
GSM678805 & MCF-7 silenced estrogen receptor, biological rep1 & 4.47218 \\
GSM678806 & MCF-7 silenced estrogen receptor, biological rep2 & 4.38426 \\
GSM678807 & MCF-7 silenced estrogen receptor, biological rep3 & 4.34567
\end{tabular}

adj.P.Val $=0.00000000000329$

Value: $m R N A$ expression level.
TABLE 3 | DNAJC12 mRNA expression in gene chip (E-GEOD-27473).

\begin{tabular}{llc}
\hline Sample & \multicolumn{1}{c}{ Title } & Value \\
\hline GSM678802 & MCF-7, biological rep1 & 8.71679 \\
GSM678803 & MCF-7, biological rep2 & 8.81721 \\
GSM678804 & MCF-7, biological rep3 & 8.74445 \\
GSM678805 & MCF-7 silenced estrogen receptor, biological rep1 & 6.38893 \\
GSM678806 & MCF-7 silenced estrogen receptor, biological rep2 & 5.69465 \\
GSM678807 & MCF-7 silenced estrogen receptor, biological rep3 & 5.78474
\end{tabular}

adj.P.Val $=0.0000000987$

Value: $m R N A$ expression level.

indicating that the predicted binding sites were highly matched. MCF-7 cells were treated with ESR1 plasmid, then examined DNAJC12 expression at various times, and it was found that the expression of DNAJC12 protein was increased (Figures 2A-C). These all indicate that ESR1 is an upstream gene of DNAJC12 and can promote the expression of DNAJC12 in MCF-7 cells.

\section{DNAJC12 Enhances Expression of ERBB4}

With the help of VBA programming language and key genes of the signaling pathways provided by cBioPortal, the candidate genes were obtained, including CCNE1, APH1B, IGF1R, BCL2, and ERBB4 (Table 5), which may be related to DNAJC12 in MCF-7 cells. Then, it was found that only ERBB4 was significantly different after detecting the mRNA expression levels of these candidate genes by PCR (Figures 3A-G). Moreover, the same trend was observed at the protein level (Figures 3H-J). These results indicate that in MCF-7 cells, ERBB4 expression can be elevated by DNAJC12.

\section{ESR1 Up-regulates Expression of ERBB4}

Based on previous results in this study, a new problem was arisen that whether ESR1 was also the upstream gene of ERBB4. ERBB4 may be regulated by ESR1 according to our results showing that ESR1 can elevate the expression of DNAJC12 (Figures 2A-C) and DNAJC12 can enhance the expression of ERBB4 (Figure 3). Therefore, ERBB4 expression was detected by western blotting following transfection of ESR1-overexpressed plasmid in MCF-7 and it was shown that the expression level of ERBB4 was increased (Figures 2D, E). Furthermore, re-analysis of EGEOD-27473 noticed that the ERBB4 mRNA expression was decreased following ESR1 silencing (Table 6), which was consistent with our previous results (Figure 2D). In brief, ESR1 is an upstream gene of ERBB4 and can up-regulate the expression of ERBB4 in MCF-7 cells.

\section{Positive Correlations Among the Three Genes}

The cBioPortal tool was employed to analyze the TCGA database for correlativity among the expression of ESR1, DNAJC12, and ERBB4 in breast carcinoma. ESR1 mRNA expression was positively correlated with the mRNA expression levels of DNAJC12 and ERBB4, with Spearman rank correlation coefficients of 0.58 and 0.63 , respectively (Figures 4A, B). In addition, the mRNA expression levels of DNAJC12 and ERBB4 showed a positive correlation, and with a Spearman rank correlation coefficient of 0.55 (Figure 4C). 
TABLE 4 | Potential ESR1 binding site on the DNAJC12 promoter region.

\begin{tabular}{|c|c|c|c|c|}
\hline Factor name & Start position & End position & Dissimilarity & String \\
\hline ESR1 [T00261] & 125 & 129 & less than 0.000001 & GGTCA \\
\hline ESR1 [T00261] & 450 & 454 & less than 0.000001 & GGTCA \\
\hline ESR1 [T00261] & 886 & 890 & less than 0.000001 & TGACC \\
\hline ESR1 [T00261] & 1034 & 1038 & less than 0.000001 & TGACC \\
\hline
\end{tabular}

The base positions in the table are consistent with those in Figure $\mathbf{1}$

\section{Analysis Number of Genes Related to ESR1, DNAJC12, and ERBB4}

A new problem was arisen whether there are genes in common that correlate with ESR1, DNAJC12, and ERBB4 expression in breast carcinoma, the cBioPortal tool was first used to output the lists containing genes related to ESR1, DNAJC12, or ERBB4. Secondly, using the VBA program, we identified a number of genes from the lists and the genes were stored in three separate lists. Last, the genes from new lists were used to draw a Venn diagram by $\mathrm{R}$ language (Figure 4D). The number of common genes (i.e. the overlapping parts of the three circles) was 12,165 , accounting for $66.41 \%$, while the non-overlapping parts contained a total of 2,622 genes (including 746 genes related to ESR1, 896 genes related to DNAJC12, and 980 genes related to ERBB4), only accounting for $14.315 \%$. In addition, the total number of two circular overlapping parts was 3,530, whose total percentage was $19.272 \%$. Judging from the percentage, the number of identical genes having correlativity with ESR1, DNAJC12, and ERBB4 respectively was 12,165 , and its proportion was $66.41 \%$, which accounts for the majority of genes in the three independent lists.

\section{DISCUSSION}

These studies suggest that ESR1 and DNAJC12 may have a certain relationship in breast carcinoma. ERBB4 is a receptor tyrosine kinase that plays a very important role in the development of the mammary gland (46). Studies have shown that this protein expression is related to ESR1 (47-50), but no reports have shown that there is a regulatory relationship between ERBB4 and DNAJC12 protein.

The relation between ESR1 and DNAJC12 was explored in the study. The VBA program and the genes of signal pathways provided by cBioPortal were combined to create an Excel macro workbook to predict DNAJC12-related genes involved in multiple signaling pathways. It was shown that ESR1 and ERBB4 are upstream and downstream genes of DNAJC12, respectively. ESR1 positively correlates with DNAJC12 and ERBB4, and DNAJC12 also positively correlates with ERBB4 in breast carcinoma.

Currently, DNAJC12 has been seldom researched in breast carcinoma, and the relation between DNAJC12 and ESR1 is still unclear. Analysis of gene expression showed that when expression of ESR1 is decreased in MCF-7 cells, DNAJC12 mRNA is also decreased, similar to that found in prior research (36). DNAJC12 promoter region has potential ESR1 binding sites, suggesting DNAJC12 expression could be regulated by ESR1, which is supported by our demonstration that protein expression of DNAJC12 is increased following overexpression of ESR1. All signs point to the conclusion that ESR1 acts upstream of DNAJC12 to enhance DNAJC12 expression in breast carcinoma. However, it should be noted that there is another mechanism for regulating the expression of DNAJC12. In prostate cancer cells, the

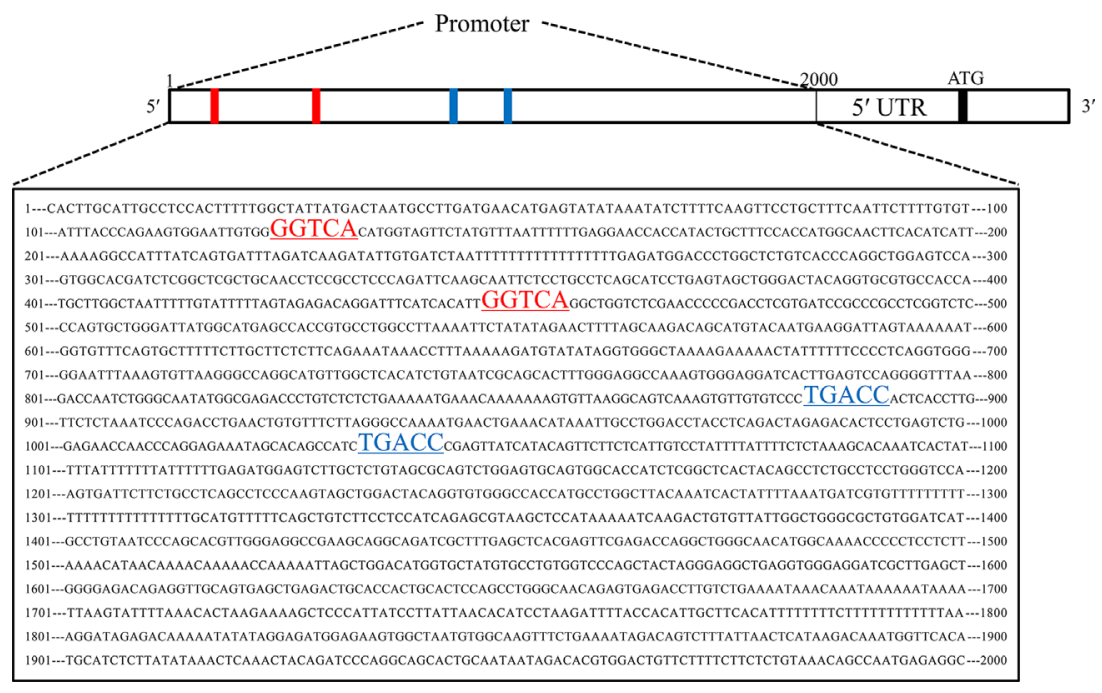

FIGURE 1 | Predicted ESR1 binding sites. The first base to the 2,000 th base is DNAJC12 promoter, the red underlined areas and blue underlined areas were both predicted ESR1 binding sites. 
A

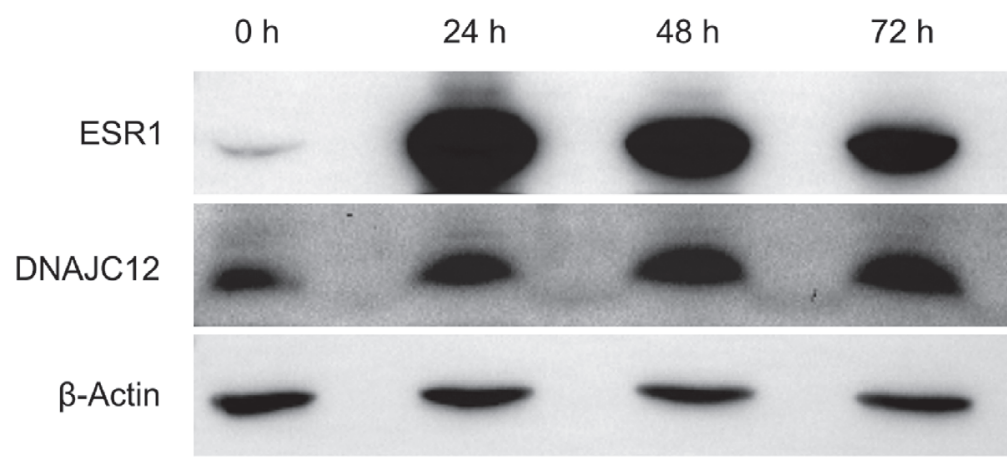

B

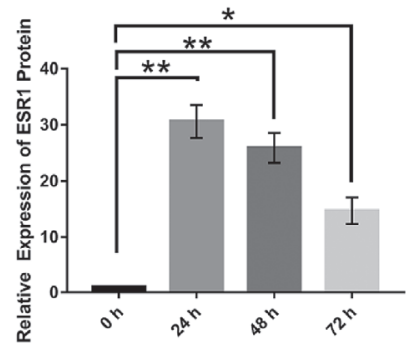

C

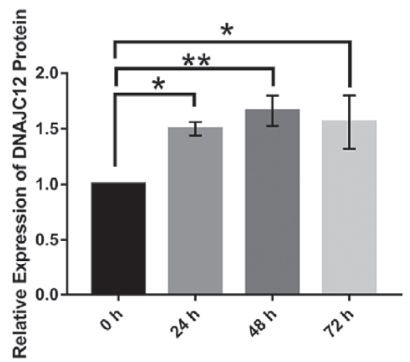

D $\mathrm{Oh}$

$24 \mathrm{~h}$

$48 \mathrm{~h}$

$72 \mathrm{~h}$

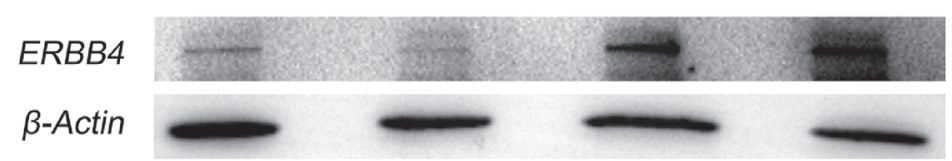

E

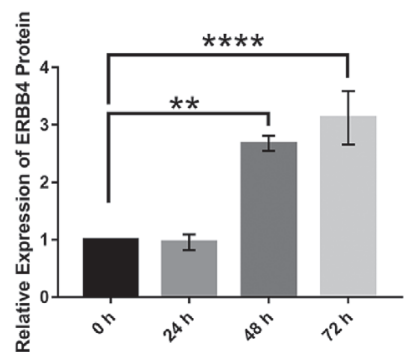

FIGURE 2 | Effect of ESR1 protein overexpression on the expression of DNAJC12 protein and ERBB4 protein. (A) MCF-7 cells were transfected with ESR1 plasmids and at 0, 24, 48, and $72 \mathrm{~h}$ after transfection, western blotting was performed to detect protein expression of ESR1 and DNAJC12 ( $\mathrm{n}=5)$. (B) Bar chart of ESR1 protein expression level. (C) Bar chart of DNAJC12 protein expression. (D) MCF-7 cells were transfected with ESR1 plasmids, and at 0, 24,48 , and $72 \mathrm{~h}$ after transfection, western blotting was performed to detect expression of ERBB4 $(n=3)$. (E) Bar chart of ERBB4 protein expression. $\left({ }^{*} P<0.05\right.$, ${ }^{* \star} P<0.01$, $\left.{ }^{* \star \star *} \mathrm{P}<0.0001\right)$.

TABLE 5 | Screening results of gene group having correlativity with DNAJC12 by VBA program.

\begin{tabular}{lccccl}
\hline Correlated Gene & Cytoband & Spearman's Correlation & $\boldsymbol{P}$-value & Q-value & Involvement in signaling regulation and control \\
\hline CCNE1 & $19 \mathrm{q} 12$ & -0.531 & $3.84 \mathrm{E}-81$ & $6.41 \mathrm{E}-79$ & Cell cycle control \\
APH1B & $15 \mathrm{q} 22.2$ & 0.583 & $2.59 \mathrm{E}-101$ & $1.94 \mathrm{E}-98$ & Notch signaling \\
IGF1R & $15 \mathrm{q} 26.3$ & 0.537 & $4.29 \mathrm{E}-83$ & $8.94 \mathrm{E}-81$ & Other growth/proliferation signaling, RTK signaling family \\
BCL2 & $18 \mathrm{q} 21.33$ & 0.603 & $1.09 \mathrm{E}-109$ & $1.38 \mathrm{E}-106$ & Survival/cell death regulation signaling \\
ERBB4 & $2 \mathrm{q} 34$ & 0.550 & $3.63 \mathrm{E}-88$ & $1.06 \mathrm{E}-85$ & RTK signaling family \\
\hline
\end{tabular}

mRNA expression of DNAJC12 can be also enhanced by AIbZIP (androgen-regulated transcription factor) located in the endoplasmic reticulum, which is relevant to the androgen and endoplasmic reticulum stress $(34,51,52)$. Thus, the expression of DNAJC12 may be regulated by more than one protein, and may also be regulated by different proteins in different cell lines. 


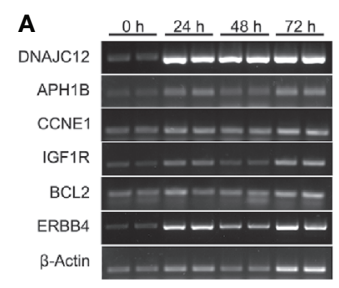

B
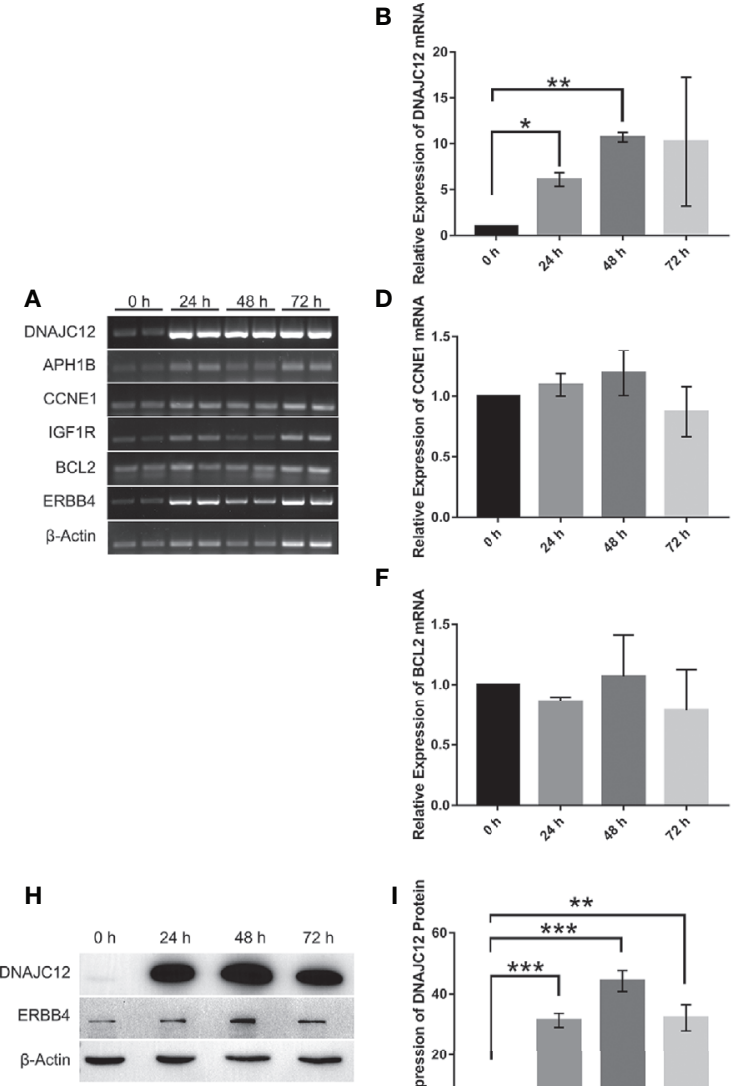
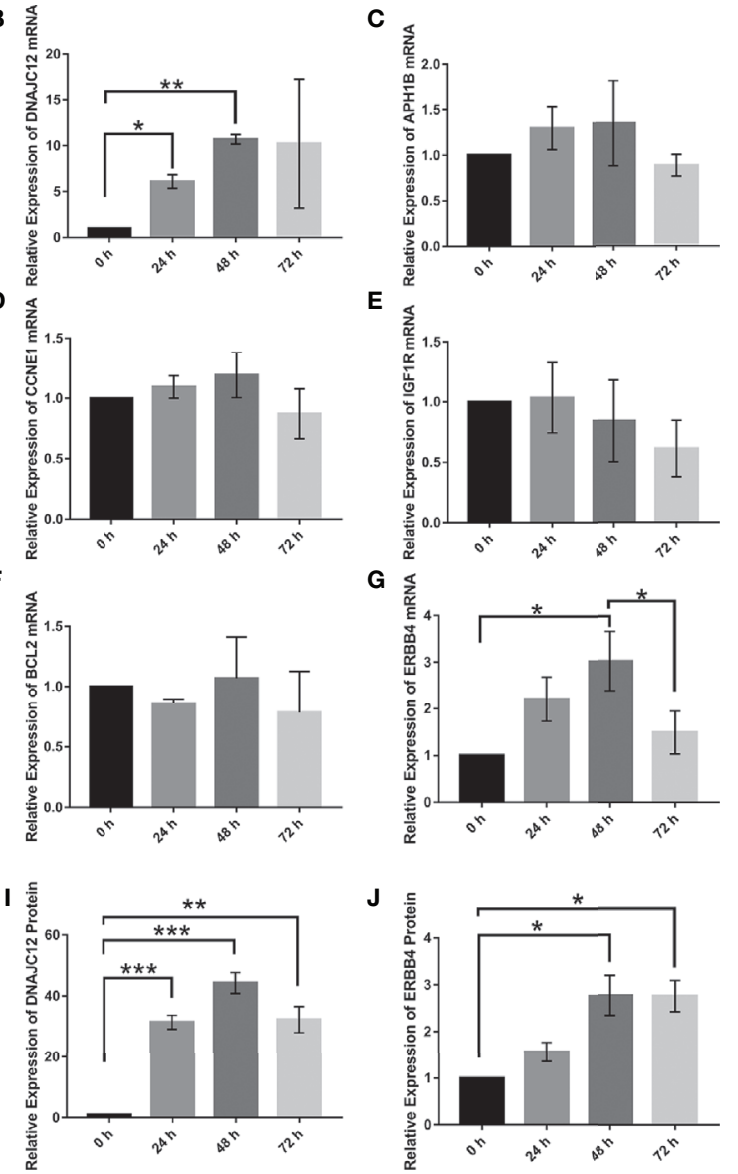

FIGURE 3 | Effect of DNAJC12 overexpression on expression of various genes. (A) MCF-7 cells were transfected with DNAJC12 plasmids, and then the mRNA of various genes was detected by PCR at 0, 24, 48, and $72 \mathrm{~h}(\mathrm{n}=3)$. (B) DNAJC12 mRNA expression. (C) APH1B mRNA expression. (D) CCNE1 mRNA expression. (E) IGF1R mRNA expression. (F) BCL2 mRNA expression. (G) ERBB4 mRNA expression. (H) MCF-7 cells were transfected with DNAJC12 plasmids, and then western blotting was used to detect the protein expression levels of DNAJC12 and ERBB4 at 0, 24, 48, and 72 h ( $\mathrm{n}=5$ ). (I) DNAJC12 protein expression. (J) ERBB4 protein expression. ( $\left.{ }^{\star} \mathrm{P}<0.05,{ }^{\star \star} \mathrm{P}<0.01,{ }^{\star \star \star} \mathrm{P}<0.001\right)$.

TABLE 6 | ERBB4 mRNA expression in gene chip (E-GEOD-27473).

\begin{tabular}{|c|c|c|}
\hline Sample & Title & Value \\
\hline GSM678802 & MCF-7, biological rep1 & 7.0086 \\
\hline GSM678803 & MCF-7, biological rep2 & 7.03895 \\
\hline GSM678804 & MCF-7, biological rep3 & 6.90234 \\
\hline GSM678805 & MCF-7 silenced estrogen receptor, biological rep1 & 4.49171 \\
\hline GSM678806 & MCF-7 silenced estrogen receptor, biological rep2 & 4.58529 \\
\hline GSM678807 & MCF-7 silenced estrogen receptor, biological rep3 & 4.37605 \\
\hline
\end{tabular}

adj.P.Val=0.00000000191.

Value: $m R N A$ expression level.

Overexpression of the carboxyl terminus sequence (CHIP) of Hsc70 in MCF-7 cells can decrease the protein level of endogenous ESR1, and furthermore attenuate ESR1-mediated gene expression (53). Also, in non-stressed $\mathrm{LNCaP}$ cells, DNAJC12 generally binds to Hsc70 (34). So, it can be speculated that if the interaction between DNAJC12 and Hsc70 in breast carcinoma cells is the same as that in LNCaP cells, DNAJC12 is bound to Hsc70, and the CHIP structure of Hsc70 is used to inhibit the expression of ESR1 protein, thus inhibiting the gene transcription mediated by ESR1. So far, the regulatory chain between ESR1 and DNAJC12 has been revealed, but the signaling pathways involving by DNAJC12 is still unclear.

In order to explore the signaling pathways associated with DNAJC12, the data from 1,108 breast carcinoma samples in the TCGA database was analyzed by using the cBioPortal tool and VBA program to obtain five candidate genes. Through a series of 
A

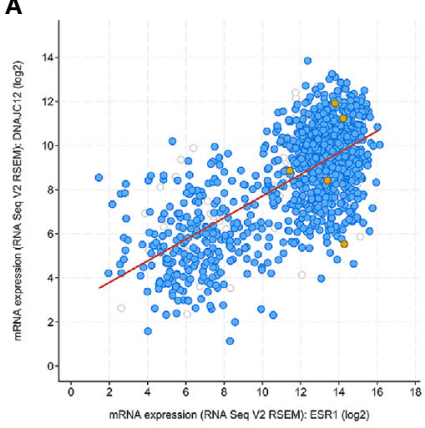

C

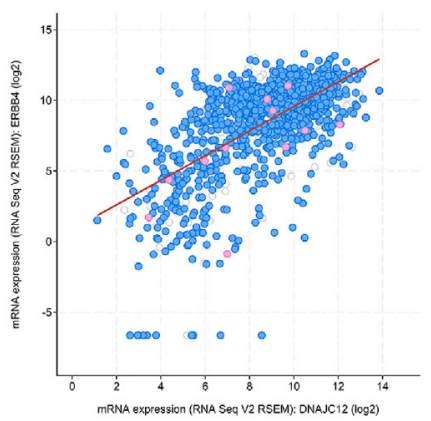

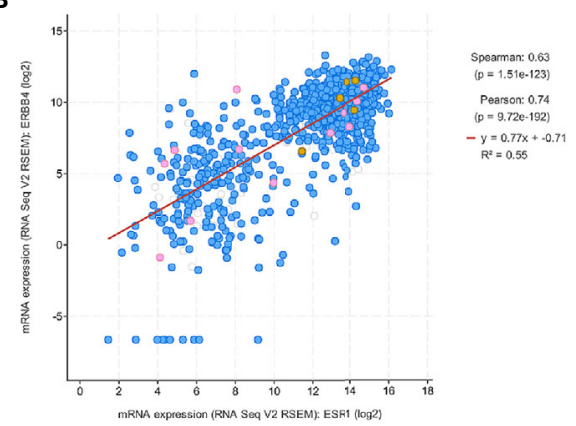

D
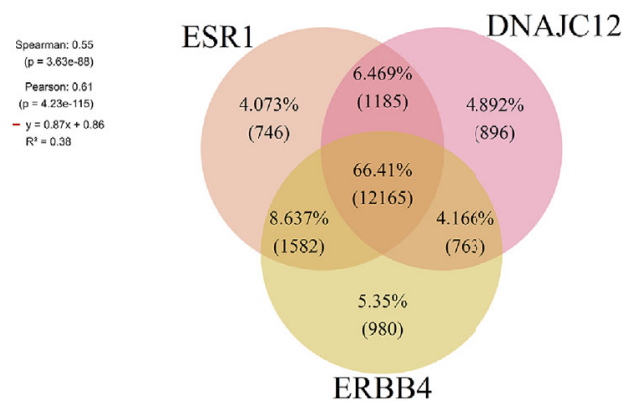

FIGURE 4 | Analysis of breast carcinoma samples from the TCGA database. (A) Relational diagram between mRNA expression levels of ESR1 and DNAJC12. (B) Relational diagram between mRNA expression levels of ESR1 and ERBB4. (C) Relational diagram between mRNA expression levels of DNAJC12 and ERBB4. (D) Venn diagram of genes having correlativity with ESR1, DNAJC12, and ERBB4. The flesh color circle represents genes having correlativity with ESR1, the pink circle represents genes having correlativity with DANJC12, and the claybank circle represents the genes having correlativity with ERBB4.

experiments, we confirmed that ERBB4 is a downstream gene of DNAJC12. Additionally, in order to determine the specific signal pathway involved in ERBB4, the KEGG database (https://www. kegg.jp/kegg/pathway.html) was queried and it was found the signal pathway involved in ERBB4 was ERBB signaling pathway (URL: https://www.kegg.jp/kegg-bin/highlight_pathway?scale= 1.0\&map $=$ map04012\&keyword=ERBB4). Thus, ERBB4 is a downstream gene of DNAJC12, and this gene is involved in the ERBB signaling pathway. However, because we did not perform co-immunoprecipitation experiments, there we do not provide direct evidence to confirm whether the association between DNAJC12 and ERBB4 is direct or indirect. And it maybe contains another mechanism, which need to be explored in the future. Hsp47 can bind to DDR2 (discoidin domain receptor tyrosine kinase 2) to DDR2 stability (24). Similar to DDR2, ERBB4 is also a tyrosine kinase. Thus, we speculate that the relationship between DNAJC12 and ERBB4 is similar that between Hsp47 and DDR2.

This research has shown that ESR1 is upstream of DNAJC12 and ERBB4 is downstream of DNAJC12, which raises the question of whether the expression of ESR1 is related to the expression of ERBB4. To test this relationship, the expression of ERBB4 in ESR1-overexpressed MCF-7 cells was examined and found that the expression level of ERBB4 protein is increased, which suggests a relationship between ERBB4 and ESR1, and explains the decrease in ERBB4 mRNA expression level in ESR1- silenced MCF-7 cells indicated by the gene expression dataset (E-GEOD-27473). Also, a positive association between ESR1 and ERBB4 is consistent with the trend shown in two patient datasets (GSE20194 and GSE23988) (49).

ERBB4 and three other tyrosine kinases, ERBB1 (epidermal growth factor receptor), ERBB2 (erb-b2 receptor tyrosine kinase 2), and ERBB3 (erb-b2 receptor tyrosine kinase 3), together form the ERBB protein family (54). In MCF-7 cells, the cytoplasmic domain 2 (CYT-2) of ERBB4 can enhance gene transcription mediated by ESR1 (48). Additionally, ERBB4 generally includes an extracellular domain, a structure near the membrane area (JM-a, rich in cysteine), a structure near the membrane area b (JM-b, rich in cysteine), transmembrane domain, tyrosine kinase domain, cytoplasmic structure 1 (CYT-1), and cytoplasmic structure 2 (CYT-2). After cleavage by TACE (tumor necrosis factor converting enzyme), the extracellular region of ERBB4 protein is released, and $\gamma$-secretase can further act on the transmembrane domain and produce soluble intracellular domain (ICD), including the tyrosine kinase domain, CYT-1, CYT-2, and other structures. The CYT-1 structure of the ICD can target the PI3K protein (55). In addition, the expression of ESR1 and ERBB4 can be inhibited by the same miRNAs (hsa -miR-28-5p, hsa-miR-665, and hsa-miR-708-5p), and thus, there may be a competitive combination that may lead to a dynamic balance between the expression of ESR1 and ERBB4 (47). MicroRNAs, including miR-375, miR-592, and miR-135a, 


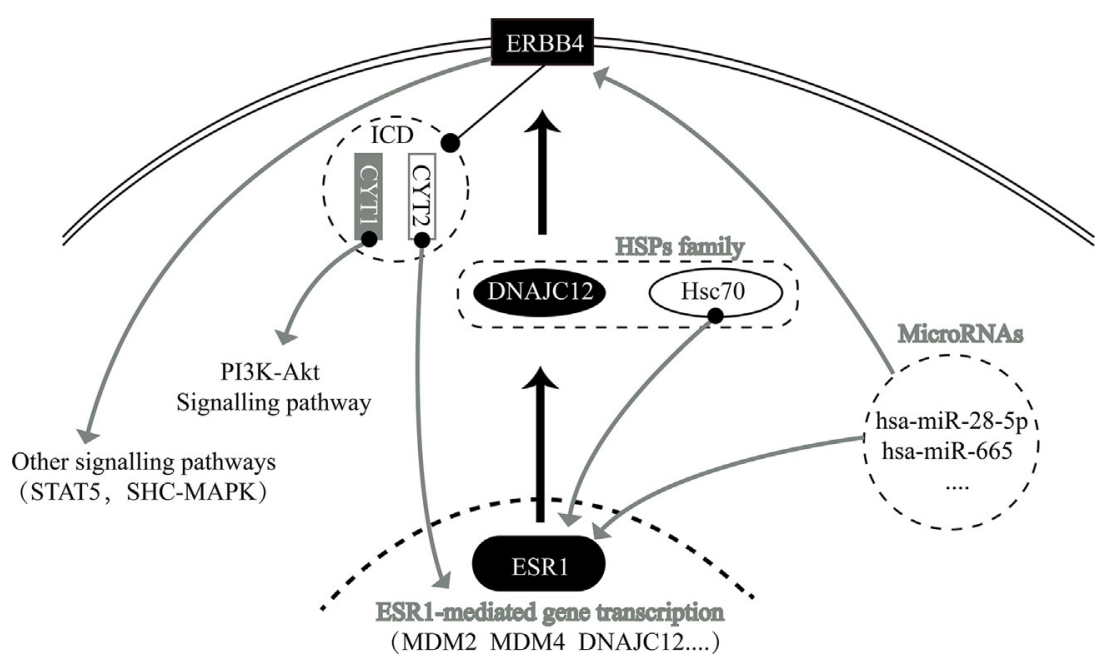

FIGURE 5 | Relationship network diagram among some genes, including ESR1, DNAJC12, and ERBB4.

can jointly inhibit the expression of ESR1 and ERBB4 (50). In short, ESR1 can promote the expression of ERBB4, and proteases can cleave ERBB4 to generate additional structures. And then, the structures can enhance gene transcription mediated by ESR1, and the expression levels of both ESR1 and ERBB4 may also be constrained by multiple miRNAs.

In addition, in order to further investigate the relationship among the expression of these three genes in breast carcinoma, the cBioPortal tool was employed to analyze the gene expression profile data in the TCGA database. In the result, ESR1 is positively correlated with DNAJC12 and ERBB4, and DNAJC12 is positively correlated with ERBB4. The trend of data distribution is similar to the trend of our cell experimental results. Based on the above analysis, ESR1, DNAJC12, and ERBB4 are found to be closely related. According to the Venn diagram, those genes which were related to these three genes occupied the majority of genes, accounting for $66.41 \%$. This suggests that not only are the three genes related to each other, but also there are many identical genes potentially related to all three genes in breast carcinoma, which imply that there may be a regulatory network among them.

In summary, we have determined that ESR1 and ERBB4 are upstream and downstream genes, respectively, of DNAJC12 in breast carcinoma. Moreover, ESR1, DNAJC12 and ERBB4 have a regulatory relationship with each other and with other genes (Figure 5). Importantly, we demonstrate the status of DNAJC12 in the ERBB signaling pathway, and also provide a new regulatory mechanism between ESR1 and ERBB4.

\section{CONCLUSIONS}

Our study shows that ESR1 and ERBB4 are the upstream and downstream genes, respectively, of DNAJC12, making ERBB4 a downstream gene of ESR1. ESR1 is positively correlated with DNAJC12 and ERBB4, and DNAJC12 is positively correlated with ERBB4 in breast carcinoma. The number of genes having correlativity with ESR1, DNAJC12, and ERBB4 is 12,165, accounting for $66.41 \%$ of the total number of genes associated with each gene individually, implicating that there may be a regulatory network among ESR1, DNAJC12, and ERBB4.

\section{DATA AVAILABILITY STATEMENT}

Publicly available data sets were analyzed in this study. These data can be found here: EMBL (https://www.ebi.ac.uk/) (EGEOD-27473) and cBioPortal (http://www.cBioPortal.org/).

\section{AUTHOR CONTRIBUTIONS}

HC and ML designed the study together, and all members performed all experiments and interpreted the results. ML, YW, and HC wrote the manuscript. All authors contributed to the article and approved the submitted version.

\section{FUNDING}

This work was supported by the Postgraduate Training Fund of Shantou University Medical College (510817044) and Guangdong Provincial Key Laboratory for Breast Cancer Diagnosis and Treatment (2017B030314116).

\section{ACKNOWLEDGMENTS}

We thank Professor Wenxiu Ni, Department of Chemistry, Shantou University Medical College for giving the MCF-7 cells to us. 


\section{REFERENCES}

1. Siegel RL, Miller KD, Jemal A. Cancer statistics, 2020. CA Cancer J Clin (2020) 70(1):7-30. doi: 10.3322/caac.21590

2. Australian Institute of Health and Welfare 2019. Cancer in Australia 2019. Cancer Ser (2019) 119:38-42. Cat. no. CAN 123. Canberra: AIHW.

3. Ghoncheh M, Pournamdar Z, Salehiniya H. Incidence and Mortality and Epidemiology of Breast Cancer in the World. Asian Pac J Cancer Prev (2016) 17(S3):43-6. doi: 10.7314/apjcp.2016.17.s3.43

4. Czajka ML, Pfeifer C. Breast Cancer Surgery. In: . StatPearls. Treasure Island (FL: StatPearls (2020).

5. Rivera S, Hannoun-Lévi JM. Hypofractionated radiation therapy for invasive breast cancer: From moderate to extreme protocols. Cancer Radiother. (2019) 23(8):874-82. doi: 10.1016/j.canrad.2019.09.002

6. Deluche E, Pierga JY. Chemotherapy and young women in breast cancer: what management? Bull Cancer (2019) 106(12S1):S19-23. doi: 10.1016/ S0007-4551(20)30043-6

7. Barchiesi G, Mazzotta M, Krasniqi E, Pizzuti L, Marinelli D, Capomolla E, et al. Neoadjuvant Endocrine Therapy in Breast Cancer: Current Knowledge and Future Perspectives. Int J Mol Sci (2020) 21(10):3528. doi: 10.3390/ ijms 21103528

8. Sugie T. Immunotherapy for metastatic breast cancer. Chin Clin Oncol (2018) 7(3):28. doi: 10.21037/cco.2018.05.05

9. Yang Z, Zhang Q, Yu L, Zhu J, Cao Y, Gao X. The signaling pathways and targets of traditional Chinese medicine and natural medicine in triplenegative breast cancer. J Ethnopharmacol (2020) 10(264):113249. doi: 10.1016/j.jep.2020.113249

10. Li JJ, Shao ZM. Endocrine therapy as adjuvant or neoadjuvant therapy for breast cancer: selecting the best agents, the timing and duration of treatment. Chin Clin Oncol (2016) 5(3):40. doi: 10.21037/cco.2016.03.24

11. Jia Y, Song Y, Dong G, Hao C, Zhao W, Li S, et al. Aberrant Regulation of RAD51 Promotes Resistance of Neoadjuvant Endocrine Therapy in ERpositive Breast Cancer. Sci Rep (2019) 9(1):12939. doi: 10.1038/s41598-01949373-w

12. Kurozumi S, Yamaguchi Y, Matsumoto H, Kurosumi M, Hayashi SI, Fujii T, et al. Utility of Ki67 labeling index, cyclin D1 expression, and ER-activity level in postmenopausal ER-positive and HER2-negative breast cancer with neoadjuvant chemo-endocrine therapy. PloS One (2019) 14(5):e0217279. doi: 10.1371/journal.pone.0217279

13. Mauriac L, Durand M, Avril A, Dilhuydy JM. Effects of primary chemotherapy in conservative treatment of breast cancer patients with operable tumors larger than $3 \mathrm{~cm}$. Results of a randomized trial in a single centre. Ann Oncol (1991) 2 (5):347-54. doi: 10.1093/oxfordjournals.annonc.a057953

14. Fisher B, Bryant J, Wolmark N, Mamounas E, Brown A, Fisher ER, et al. Effect of preoperative chemotherapy on the outcome of women with operable breast cancer. J Clin Oncol (1998) 16(8):2672-85. doi: 10.1200/JCO.1998.16.8.2672

15. Mauriac L, MacGrogan G, Avril A, Durand M, Floquet A, Debled M, et al. Neoadjuvant chemotherapy for operable breast carcinoma larger than $3 \mathrm{~cm}$ : a unicentre randomized trial with a 124-month median follow-up. Institut Bergonié Bordeaux Groupe Sein (IBBGS). Ann Oncol (1999) 10(1):47-52. doi: 10.1023/a:1008337009350

16. Wolmark N, Wang J, Mamounas E, Bryant J, Fisher B. Preoperative chemotherapy in patients with operable breast cancer: nine-year results from National Surgical Adjuvant Breast and Bowel Project B-18. J Natl Cancer Inst Monogr (2001) 30:96-102. doi: 10.1093/oxfordjournals.jncimonographs.a003469

17. Suman VJ, Ellis MJ, Ma CX. The ALTERNATE trial: assessing a biomarker driven strategy for the treatment of post-menopausal women with ER+/Her2invasive breast cancer. Chin Clin Oncol (2015) 4(3):34. doi: 10.3978/ j.issn.2304-3865.2015.09.01

18. Harbeck N, Penault-Llorca F, Cortes J, Gnant M, Houssami N, Poortmans P, et al. Breast cancer. Nat Rev Dis Primers (2019) 5(1):66. doi: 10.1038/s41572019-0111-2

19. Wu J, Liu T, Rios Z, Mei Q, Lin X, Cao S. Heat Shock Proteins and Cancer. Trends Pharmacol Sci (2017) 38(3):226-56. doi: 10.1016/j.tips.2016.11.009

20. Zoppino FCM, Guerrero-Gimenez ME, Castro GN, Ciocca DR. Comprehensive transcriptomic analysis of heat shock proteins in the molecular subtypes of human breast cancer. BMC Cancer (2018) 18(1):700. doi: 10.1186/s12885-018-4621-1
21. Zhu J, Xiong G, Fu H, Evers BM, Zhou BP, Xu R. Chaperone Hsp47 Drives Malignant Growth and Invasion by Modulating an ECM Gene Network. Cancer Res (2015) 75(8):1580-91. doi: 10.1158/0008-5472.CAN-14-1027

22. Liu X, Feng C, Liu J, Cao L, Xiang G, Liu F, et al. Androgen receptor and heat shock protein 27 co-regulate the malignant potential of molecular apocrine breast cancer. J Exp Clin Cancer Res (2018) 37(1):90. doi: 10.1186/s13046-018-0762-y

23. Shao H, Li X, Moses MA, Gilbert LA, Kalyanaraman C, Young ZT, et al. Exploration of Benzothiazole Rhodacyanines as Allosteric Inhibitors of Protein-Protein Interactions with Heat Shock Protein 70 (Hsp70). J Med Chem (2018) 61(14):6163-77. doi: 10.1021/acs.jmedchem.8b00583

24. Chen J, Wang S, Zhang Z, Richards CI, Xu R. Heat shock protein 47 (HSP47) binds to discoidin domain-containing receptor 2 (DDR2) and regulates its protein stability. J Biol Chem (2019) 294(45):16846-54. doi: 10.1074/ jbc.RA119.009312

25. Xiong X, Wang Y, Liu C, Lu Q, Liu T, Chen G, et al. Heat shock protein $90 \beta$ stabilizes focal adhesion kinase and enhances cell migration and invasion in breast cancer cells. Exp Cell Res (2014) 326(1):78-89. doi: 10.1016/ j.yexcr.2014.05.018

26. Piccolella M, Crippa V, Cristofani R, Rusmini P, Galbiati M, Cicardi ME, et al. The small heat shock protein B8 (HSPB8) modulates proliferation and migration of breast cancer cells. Oncotarget (2017) 8(6):10400-15. doi: 10.18632/oncotarget.14422

27. Gomez-Monterrey I, Campiglia P, Scognamiglio I, Vanacore D, Dicitore A, Lombardi A, et al. DTNQ-Pro, a Mimetic Dipeptide, Sensitizes Human Colon Cancer Cells to 5-Fluorouracil Treatment. J Amino Acids (2013) 2013:509056. doi: $10.1155 / 2013 / 509056$

28. Gibert B, Hadchity E, Czekalla A, Aloy MT, Colas P, Rodriguez-Lafrasse C, et al. Inhibition of heat shock protein 27 (HspB1) tumorigenic functions by peptide aptamers. Oncogene (2011) 30(34):3672-81. doi: 10.1038/onc.2011.73

29. Sharma A, Upadhyay AK, Bhat MK. Inhibition of Hsp27 and Hsp40 potentiates 5-fluorouracil and carboplatin mediated cell killing in hepatoma cells. Cancer Biol Ther (2009) 8(22):2106-13. doi: 10.4161/cbt.8.22.9687

30. Pace A, Barone G, Lauria A, Martorana A, Piccionello AP, Pierro P, et al. Hsp60, a novel target for antitumor therapy: structure-function features and prospective drugs design. Curr Pharm Des (2013) 19(15):2757-64. doi: $10.2174 / 1381612811319150011$

31. Wang X, Chen M, Zhou J, Zhang X. HSP27, 70 and 90, anti-apoptotic proteins, in clinical cancer therapy (Review). Int J Oncol (2014) 45(1):18-30. doi: 10.3892/ijo.2014.2399

32. Kang BH, Plescia J, Song HY, Meli M, Colombo G, Beebe K, et al. Combinatorial drug design targeting multiple cancer signaling networks controlled by mitochondrial Hsp90. J Clin Invest (2009) 119(3):454-64. doi: 10.1172/JCI37613

33. Uno Y, Kanda M, Miwa T, Umeda S, Tanaka H, Tanaka C, et al. Increased Expression of DNAJC12 is Associated with Aggressive Phenotype of Gastric Cancer. Ann Surg Oncol (2019) 26(3):836-44. doi: 10.1245/s10434-01807149-y

34. Choi J, Djebbar S, Fournier A, Labrie C. The co-chaperone DNAJC12 binds to $\mathrm{Hsc70}$ and is upregulated by endoplasmic reticulum stress. Cell Stress Chaperones (2014) 19(3):439-46. doi: 10.1007/s12192-013-0471-6

35. Bubnov V, Moskalev E, Petrovskiy Y, Bauer A, Hoheisel J, Zaporozhan V. Hypermethylation of TUSC5 genes in breast cancer tissue. Exp Oncol (2012) 34(4):370-2.

36. De Bessa SA, Salaorni S, Patrão DF, Neto MM, Brentani MM, Nagai MA. JDP1 (DNAJC12/Hsp40) expression in breast cancer and its association with estrogen receptor status. Int J Mol Med (2006) 17(2):363-7. doi: 10.3892/ ijmm.17.2.363

37. Caligiuri I, Toffoli G, Giordano A, Rizzolio F. pRb controls estrogen receptor alpha protein stability and activity. Oncotarget (2013) 4(6):875-83. doi: 10.18632/oncotarget.1036

38. Dhamad AE, Zhou Z, Zhou J, Du Y. Systematic Proteomic Identification of the Heat Shock Proteins (Hsp) that Interact with Estrogen Receptor Alpha $(\mathrm{ER} \alpha)$ and Biochemical Characterization of the ER $\alpha-\mathrm{Hsp} 70$ Interaction. PloS One (2016) 11(8):e0160312. doi: 10.1371/journal.pone.0160312

39. Mo XM, Li L, Zhu P, Dai YJ, Zhao TT, Liao LY, et al. Up-regulation of Hsp27 by $\mathrm{ER} \alpha / \mathrm{Sp} 1$ facilitates proliferation and confers resistance to apoptosis in human papillary thyroid cancer cells. Mol Cell Endocrinol (2016) 15(431):7187. doi: 10.1016/j.mce.2016.05.010 
40. Al Saleh S, Al Mulla F, Luqmani YA. Estrogen receptor silencing induces epithelial to mesenchymal transition in human breast cancer cells. PloS One (2011) 6(6):e20610. doi: 10.1371/journal.pone.0020610

41. Messeguer X, Escudero R, Farré D, Núñez O, Martínez J, Albà MM. PROMO: detection of known transcription regulatory elements using species-tailored searches. Bioinformatics (2002) 18(2):333-4. doi: 10.1093/bioinformatics/ 18.2.333

42. Farré D, Roset R, Huerta M, Adsuara JE, Roselló L, Albà MM, et al. Identification of patterns in biological sequences at the ALGGEN server: PROMO and MALGEN. Nucleic Acids Res (2003) 31(13):3651-3. doi: 10.1093/nar/gkg605

43. Gao J, Aksoy BA, Dogrusoz U, Dresdner G, Gross B, Sumer SO, et al. Integrative analysis of complex cancer genomics and clinical profiles using the cBioPortal. Sci Signal (2013) 6(269):pl1. doi: 10.1126/scisignal.2004088

44. Cerami E, Gao J, Dogrusoz U, Gross BE, Sumer SO, Aksoy BA, et al. The cBio cancer genomics portal: an open platform for exploring multidimensional cancer genomics data. Cancer Discovery (2012) 2(5):401-4. doi: 10.1158/21598290.CD-12-0095

45. Green MJ. In-vitro antimicrobial susceptibility of Neisseria gonorrhoeae in New Zealand. Br J Vener Dis (1978) 54(5):316-21. doi: 10.1136/sti.54.5.316

46. Veikkolainen V, Vaparanta K, Halkilahti K, Iljin K, Sundvall M, Elenius K. Function of ERBB4 is determined by alternative splicing. Cell Cycle (2011) 10 (16):2647-57. doi: 10.4161/cc.10.16.17194

47. Bagheri F, Mesrian Tanha H, Mojtabavi Naeini M, Ghaedi K, Azadeh M. Tumor-promoting function of single nucleotide polymorphism rs1836724 (C3388T) alters multiple potential legitimate microRNA binding sites at the 3'-untranslated region of ErbB4 in breast cancer. Mol Med Rep (2016) 13 (5):4494-8. doi: 10.3892/mmr.2016.5078

48. Junttila TT, Sundvall M, Lundin M, Lundin J, Tanner M, Härkönen P, et al. Cleavable ErbB4 isoform in estrogen receptor-regulated growth of breast cancer cells. Cancer Res (2005) 65(4):1384-93. doi: 10.1158/0008-5472.CAN-04-3150

49. Shao N, Yuan K, Zhang Y, Yun Cheang T, Li J, Lin Y. Identification of key candidate genes, pathways and related prognostic values in ER-negative/ HER2-negative breast cancer by bioinformatics analysis. J BUON (2018) 23 (4):891-901.
50. Yang Y, Xing Y, Liang C, Hu L, Xu F, Chen Y. Crucial microRNAs and genes of human primary breast cancer explored by microRNA-mRNA integrated analysis. Tumour Biol (2015) 36(7):5571-9. doi: 10.1007/s13277-015-3227-3

51. Ben Aicha S, Lessard J, Pelletier M, Fournier A, Calvo E, Labrie C. Transcriptional profiling of genes that are regulated by the endoplasmic reticulum-bound transcription factor AIbZIP/CREB3L4 in prostate cells. Physiol Genomics (2007) 31(2):295-305. doi: 10.1152/physiolgenomics.00097.2007

52. Qi H, Fillion C, Labrie Y, Grenier J, Fournier A, Berger L, et al. AIbZIP, a novel bZIP gene located on chromosome 1q21.3 that is highly expressed in prostate tumors and of which the expression is up-regulated by androgens in $\mathrm{LNCaP}$ human prostate cancer cells. Cancer Res (2002) 62(3):721-33.

53. Fan M, Park A, Nephew KP. CHIP (carboxyl terminus of Hsc70-interacting protein) promotes basal and geldanamycin-induced degradation of estrogen receptor-alpha. Mol Endocrinol (2005) 19(12):2901-14. doi: 10.1210/me.20050111

54. Yamamoto H, Higa-Nakamine S, Noguchi N, Maeda N, Kondo Y, Toku S, et al. Desensitization by different strategies of epidermal growth factor receptor and ErbB4. J Pharmacol Sci (2014) 124(3):287-93. doi: 10.1254/ jphs.13r11cp

55. Määttä JA, Sundvall M, Junttila TT, Peri L, Laine VJ, Isola J, et al. Proteolytic cleavage and phosphorylation of a tumor-associated ErbB4 isoform promote ligand-independent survival and cancer cell growth. Mol Biol Cell (2006) 17 (1):67-79. doi: 10.1091/mbc.e05-05-0402

Conflict of Interest: The authors declare that the research was conducted in the absence of any commercial or financial relationships that could be construed as a potential conflict of interest.

Copyright (c) 2021 Lin, Wang, Ye, Xiong, Guo and Chen. This is an open-access article distributed under the terms of the Creative Commons Attribution License (CC BY). The use, distribution or reproduction in other forums is permitted, provided the original author(s) and the copyright owner(s) are credited and that the original publication in this journal is cited, in accordance with accepted academic practice. No use, distribution or reproduction is permitted which does not comply with these terms. 